Article

\title{
Flutter Analysis of a Transonic Steam Turbine Blade with Frequency and Time-Domain Solvers ${ }^{+}$
}

\author{
Christian Frey *, Graham Ashcroft, Hans-Peter Kersken and Daniel Schlüß \\ Institute of Propulsion Technology, German Aerospace Center (DLR), Linder Höhe, 51147 Köln, Germany; \\ Graham.Ashcroft@dlr.de (G.A.); Hans-Peter.Kersken@dlr.de (H.-P.K.); Daniel.Schluess@dlr.de (D.S.) \\ * Correspondence: Christian.Frey@dlr.de \\ t This paper is an extended version of our paper in Proceedings of the 13th European Turbomachinery \\ Conference on Fluid Dynamics \& Thermodynamics ETC13, Lausanne, Switzerland, 8-12 April 2019. \\ Paper No. 32.
}

Received: 10 May 2019; Accepted: 17 May 2019; Published: 12 June 2019

\begin{abstract}
The aim of this study was to assess the capabilities of different simulation approaches to predict the flutter stability of a steam turbine rotor. The focus here was on linear and nonlinear frequency domain solvers in combination with the energy method, which is widely used for the prediction of flutter onset. Whereas a GMRES solver was used for the linear problem, the nonlinear methods employed a time-marching procedure. The solvers were applied to the flutter analysis of the first rotor bending mode of the open Durham Steam Turbine test case. This test case is representative of the last stage of modern industrial steam turbines. We compared our results to those published by other researchers in terms of aerodynamic damping and local work per cycle coefficients. Time-domain, harmonic balance, and time-linearised methods were compared to each other in terms of CPU efficiency and accuracy.
\end{abstract}

Keywords: steam turbine; flutter; transonic flows; frequency-domain solvers

\section{Introduction}

The efficient and accurate prediction of turbine flutter during the design phase is vital since flutter stability and aerodynamic efficiency are often competing goals. As the onset of flutter is a phenomenon which is explained by the instability of the fluid-structure system at rest, flutter predictions are often performed with linearised solvers in the frequency domain. There is, however, still a need for aeroeleastic analysis tools with higher accuracy and the capability to resolve nonlinear unsteady flow phenomena. Firstly, nonlinearities may be important for forced response applications or interactions with flow instabilities, i.e., situations where the vibration amplitude can no longer be considered as infinitesimal. Secondly, for transonic test cases, shock boundary layer interactions may result in local nonlinear responses of the unsteady flow, even if the overall work per cycle depends almost linearly on the vibration amplitude. Here, unsteady turbulence modelling can be crucial to obtain physically meaningful results [1]. For cases with periodic shock oscillations, linear solvers may produce unphysically high pressure amplitudes which depend strongly on the mesh resolution and the spatial discretisation. Although, in this case, the CFD result can be a good approximation in terms of the overall aerodynamic damping [2], it is very difficult to compare local unsteady pressures with experimental data, for instance from pressure transducers [3,4].

For applications where the unsteadiness is aperiodic or where the frequency is unknown, it is important to have an efficient and accurate time-domain simulation method that can handle blade vibrations. One of the difficulties with time-domain simulations is that they often rely on less accurate inlet and outlet boundary conditions than their frequency domain counterparts. Since numerical 
reflections can be particularly detrimental for aeroelastic simulations, the authors made great efforts to develop robust frequency-domain nonreflecting boundary conditions for the time-domain solver [5].

In this study, the methods above were applied to the flutter analysis of an open access steam turbine [6] established by Qi et al. [7] as an open flutter test case. We demonstrated the applicability of the approaches and compared the results to each other and to results obtained by Qi et al. [7] and Sun et al. [8] with other flow solvers. Note that Fuhrer and Vogt [9] also applied a variety of simulation approaches to predict the aerodynamic damping of this turbine rotor. Their setup, however, differs from ours in the reduced frequency (ours is that suggested by Qi et al.). Moreover, Fuhrer and Vogt modified the blade count to match the vane count.

\section{Numerical Methods}

The methods described below are integrated into the DLR flow solver TRACE. They can be viewed as different numerical approaches to solve the semi-discrete equations

$$
\frac{\partial}{\partial t}(V q)+V R(q, x, \dot{x})=0 .
$$

Since we compared unsteady flows on moving meshes, we stressed the dependency of the residual $R$ on the grid coordinates $x$ and their velocities $\dot{x} . q, V$, and $R$ denote the conservative flow vector, the time-dependent cell volume, and the flow residual, respectively. The underlying spatial discretisation employed in this study was based on the finite volume approach. The study in this work was carried out with structured grids, on which we used Roe's upwind scheme [10] in combination with a MUSCL extrapolation to achieve second-order accuracy [11]. A van Albada type limiter was employed to smoothen the solutions in the vicinity of shocks [12]. Harten's entropy fix [13] with a parameter of $\varepsilon=5 \times 10^{-4}$ was used.

TRACE has three approaches to simulate the unsteady Reynolds-averaged Navier-Stokes (URANS) equations: the time-domain, the harmonic balance, and the time-linearised solver [14,15]. The time-domain solver uses dual-time stepping in combination with a backward differencing or implicit Runge-Kutta schemes [16] to integrate Equation (1). The harmonic balance solver solves the URANS equations, cast in the frequency domain,

$$
\mathrm{i} \omega \widehat{V q})_{\omega}+(\widehat{V R(q)})_{\omega}=0 .
$$

The Fourier harmonic of the residual is computed in an alternating-frequency-time domain (AFT) framework $[15,17]$, i.e.,

$$
(\widehat{V R(q}))=\mathscr{F}\left(V R\left(\mathscr{F}^{-1} q\right)\right),
$$

where $\mathscr{F}$ and $\mathscr{F}^{-1}$ denote the discrete Fourier transform and its inverse. In the linearised solver [14], this expression above is approximated by

$$
\left.(\widehat{V R(q)})_{\omega} \approx \widehat{V}_{0} \widehat{R(q)}\right)_{\omega} \approx \widehat{V}_{0}\left(\left.\frac{\partial R}{\partial q}\right|_{\hat{q}_{0}} \widehat{q}_{\omega}+\frac{\partial R}{\partial x} \hat{x}+\mathrm{i} \omega \frac{\partial R}{\partial \dot{x}} \hat{x}\right),
$$

where one assumes that the amplitude of the disturbance $\hat{x}$ is small and that the mean flow $\hat{q}_{0}$ solves the steady equations $R\left(\hat{q}_{0}\right)=0$. Whereas both the time-domain and harmonic balance solvers use pseudo-time stepping, the linear solver employs a preconditioned GMRES method. In this work, the multi-step SSOR preconditioner with seven steps and a relaxation factor of 0.3 was used.

In this work, the steady and time-domain solvers were run in combination with Wilcox' twoequation turbulence model [18]. In the harmonic balance solver, resolving the unsteadiness of turbulence quantities by higher harmonics is optional [19]. The linearised solver is always based on the frozen turbulence assumption, i.e., the eddy viscosity is simply taken as the one from the steady solution. 
It is well known that, in numerical flutter and forced response analyses, modal forces are highly susceptible to spurious reflections at inlet and outlet boundaries. Therefore, the time-domain boundary conditions in TRACE were extended to a two-dimensional nonreflecting frequency-domain formulation, which is consistent with the implementations in the HB and linearised solvers [20]. In a recent effort, the authors improved the robustness and convergence speed of the nonreflecting boundary conditions (NRBCs) in the time-domain solver [5]. It should be emphasised that this formulation is also consistent with the steady flow boundary conditions. More precisely, it is guaranteed that the time mean of a converged periodic flow satisfies the steady NRBCs [21].

\section{Test Case}

The test case studied here is the design of a last stage steam turbine. The geometry is due to Burton [6] and comprises a turbine stage along with an exhaust hood. The rotor blade count was 65 with a blade length of $0.92 \mathrm{~m}$. The rotational speed was $3000 \mathrm{~min}^{-1}$. In this work, the rotor tip gap height was $2.1 \mathrm{~mm}$ (approximately $0.25 \%$ blade height), which was half the tip gap size in [6] but similar to the tip gap height of $2.3 \mathrm{~mm}$ that was used by Qi et al. [7] and Sun et al. [8].

The boundary conditions for the configuration including stator and exhaust hood were taken from [7]. The stator inlet total pressure and temperature were set to $26.7 \mathrm{kPa}$ and $339.5 \mathrm{~K}$, respectively. At the outlet, a pressure distribution satisfying the radial equilibrium equation with circumferentially mixed-out static pressure of $8.8 \mathrm{kPa}$ at midspan was used. The average isentropic exit Mach number was about 1.12 .

The test case was run with an ideal gas with the parameters listed in Table 1. For a justification of the use of ideal gas for the flutter analysis of steam turbines, the reader is referred to [22].

Table 1. Ideal gas parameters used for the steam turbine.

\begin{tabular}{l|ll}
\hline$R$ & 461.52 & $\mathrm{~J} \cdot \mathrm{kg}^{-1} \cdot \mathrm{K}^{-1}$ \\
$\gamma$ & 1.12 & - \\
$\mu$ & $1.032 \times 10^{-5}$ & $\mathrm{~N} \cdot \mathrm{s} \cdot \mathrm{m}^{-2}$ \\
\hline
\end{tabular}

For the flutter analysis, the computational domain consisted of the rotor only, i.e., both the stator and the exhaust were neglected (see Figure 1). Where necessary (time-domain and harmonic balance simulations), the radial distributions of inlet and outlet boundary values for the rotor domain were extracted from the steady simulation of the whole configuration. The block structured mesh was generated with an in-house mesh generator [23] and consisted of 4 million cells for the whole configuration and 2.1 million cells for the rotor domain alone. Blade wall boundary layers were resolved with $y^{+} \approx 1$, whereas wall functions were used for the hub and casing wall. The mesh had 88 cells from hub to casing. The tip clearance region was resolved with 10 cells in the radial direction. Mesh details are depicted in Figure 2. The relative channel height definition is adopted from [8] (see Figure 1).

The shock system predicted by the steady solver is shown in Figure 3. The comparison with results obtained with the flow solver LUFT [24] is shown for the blade pressure at $50 \%$ and $90 \%$ height in Figure 4. The TRACE results show a stronger leading edge suction peak and subsequent shock. The same holds for the pressure rise at about half chord. The authors suspect that these differences stem from the fact that the mesh used in this work was slightly finer (roughly twice as many cells in the rotor domain) than that used for LUFT. The rotor outflow was transonic almost over the whole channel height with maximal Mach numbers of about 1.5 near the casing. The Reynolds number, based on midspan chord length and the mean flow at the rotor outlet, was about 415,000.

The blade eigenmode studied here is taken from [7]. Unless otherwise stated, the maximum amplitude of the blade vibration was set to $1.5 \mathrm{~mm}$. The frequency was $132.08 \mathrm{~Hz}$, corresponding to a reduced frequency $\omega^{*}=\omega c_{\mathrm{m}} / v_{\text {out }}$ of 0.3 . 

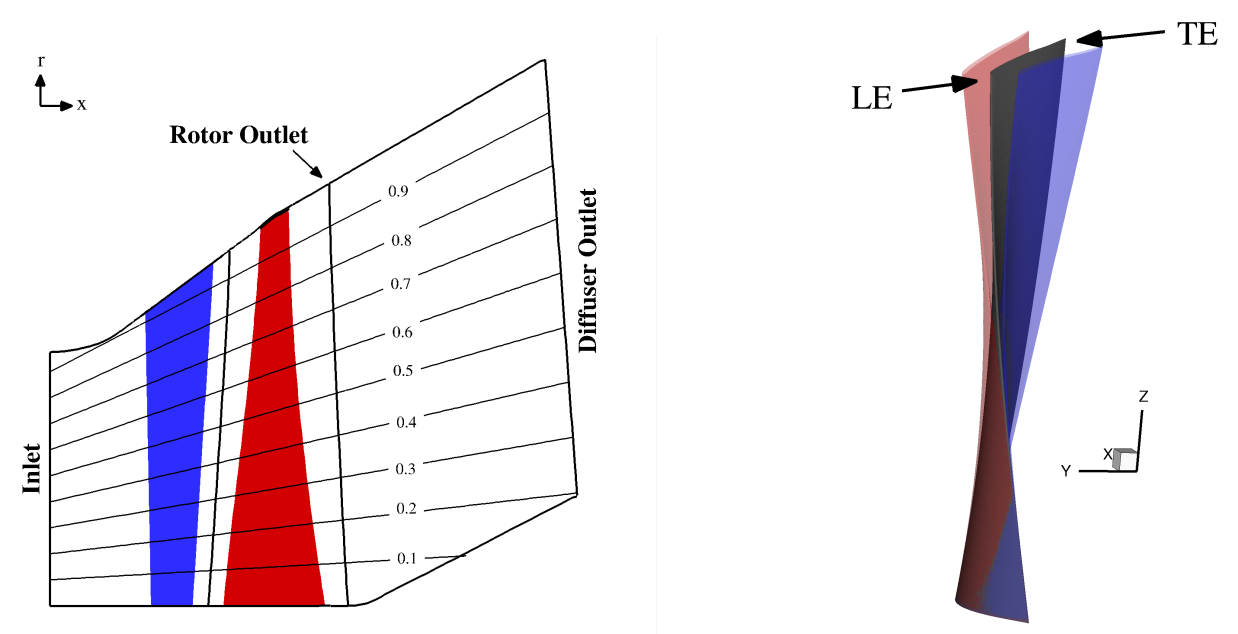

Figure 1. Sketch of the configuration in S2 plane (left) and first rotor bending mode (right).

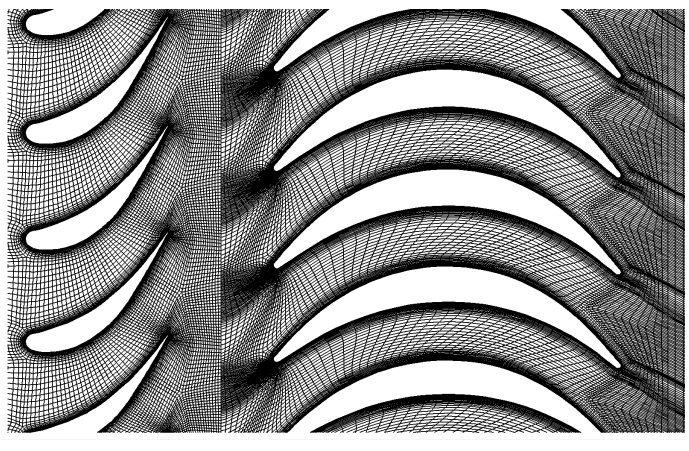

(a) Hub

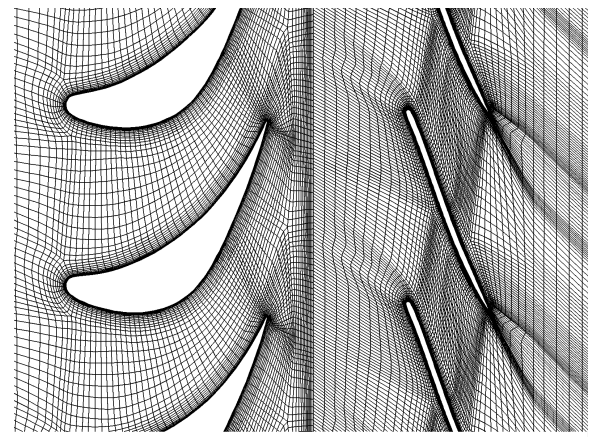

(b) Casing

Figure 2. Mesh resolution near hub and casing. Every other grid line is shown.

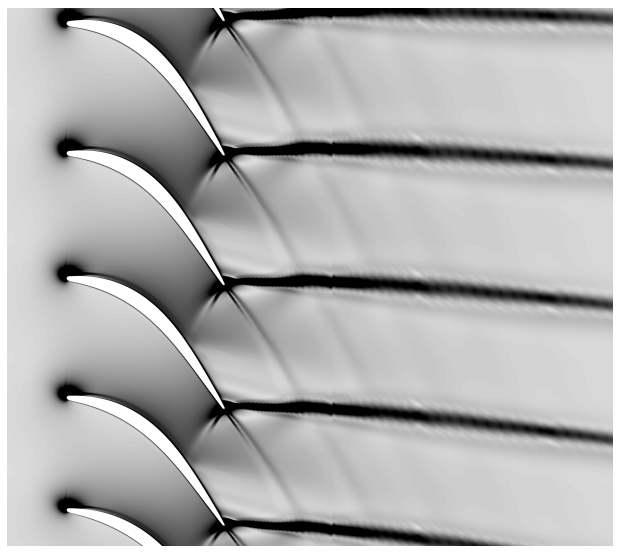

(a) $50 \%$

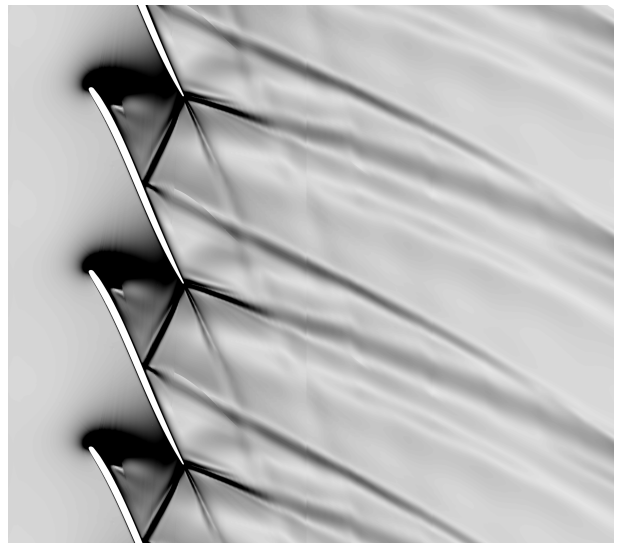

(b) $90 \%$

Figure 3. Steady solution (numerical Schlieren) in the rotor domain at 50\% and $90 \%$ channel height. 


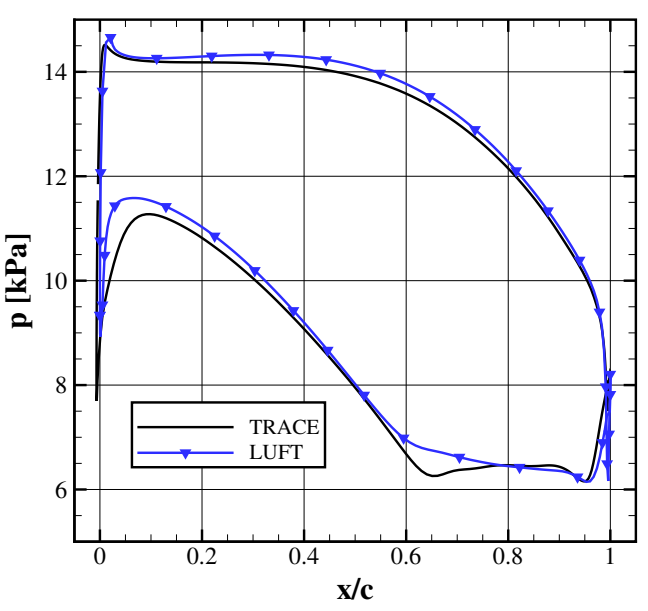

(a) $50 \%$

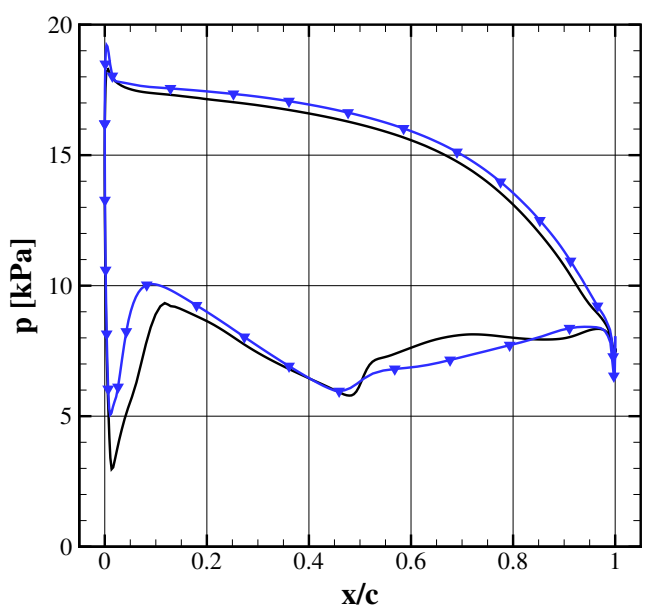

(b) $90 \%$

Figure 4. Blade pressure along $50 \%$ and $90 \%$ channel height.

\section{Results}

\subsection{Linear Solver Set-Up and Results}

The linear solver was used to compute the aerodynamic damping coefficient for all 65 nodal diameters.

First, a termination criterion was determined by comparing the residual drop to the relative variation of the aerodynamic work per cycle for the interblade phase angle $\sigma=0^{\circ}$ (see Figure 5). In this plot, the relative error was computed with respect to the reference solution after a residual drop of $10^{-4}$. Since the relative error in aerodynamic damping was below $10^{-3}$ when the $L^{2}$ residual was below $10^{-3}$, this was taken as a convergence criterion for the damping curve. For all but three interblade phase angles, the convergence speed was comparable with a total time to convergence between 77 and 140 CPUh. Exceptionally long run times (up to 622 CPUh) were observed for the interblade phase angles near $-16.6^{\circ}$. An acoustic cut-on/cut-off analysis showed that, for a large range of radii, this value was close to an acoustic resonance as predicted by a 2D modal analysis. Note that all solvers used a regularised modal decomposition to stabilise the nonreflecting boundary conditions near acoustic resonance [25].

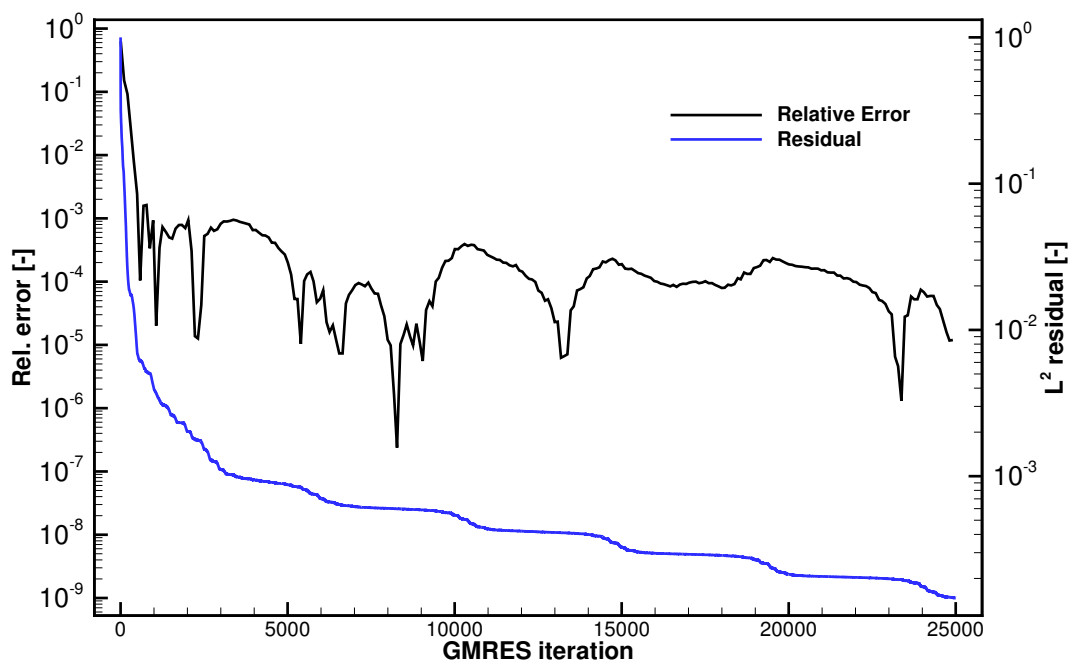

Figure 5. Relative error of work coefficient and $L^{2}$ residual over GMRES iteration for $\sigma=0^{\circ}$. 
The linear solver results are plotted in Figure 6 in terms of the aerodynamic work coefficient

$$
\Xi=-\frac{\operatorname{Re} W_{\mathrm{cyc}}}{\pi b \alpha^{2} c_{\mathrm{m}}^{2} p_{\mathrm{dyn}, \mathrm{in}}}
$$

where $\alpha=d_{\max } / c_{\mathrm{m}}$ is the non-dimensionalised amplitude and Re $W_{\text {cyc }}$ is the active aerodynamic work [14]. The dynamic pressure was computed from the mass-averaged relative stagnation and static pressures at the stator-rotor interface, which yielded $p_{\text {dyn, in }}=2127.7 \mathrm{~Pa}$. The results were compared with those obtained by Sun et al. [8] with the linearised solver LUFT [26] and the commercial software ANSYS CFX. Note that the reference data computed with LUFT and the results termed "CFX (no tip gap)" were obtained on a configuration without tip gap. As mentioned above, the configuration "CFX (tip gap)" had a tip gap size which was comparable to ours. The CFX simulations were performed in the time-domain on multi-passage grids to circumvent non-zero interblade phase angles at the perodic boundaries of the domain. The overall agreement between the linearised method in TRACE and LUFT was satisfactory. Discrepancies, however, can be seen for higher nodal diameters, in particular for interblade phase angles above $+80^{\circ}$. Acoustic resonance could be discerned near $-16.6^{\circ}$ and in the vicinity of $+22.2^{\circ}$, where for the latter region no reduction in convergence speed was observed. The location of the minimal damping predicted by linearTRACE was near $\sigma=-72^{\circ}$, which was very close to the minimum predicted by LUFT. The remainder of this section focuses on this interblade phase angle and compares the results with those obtained with the other simulation approaches.

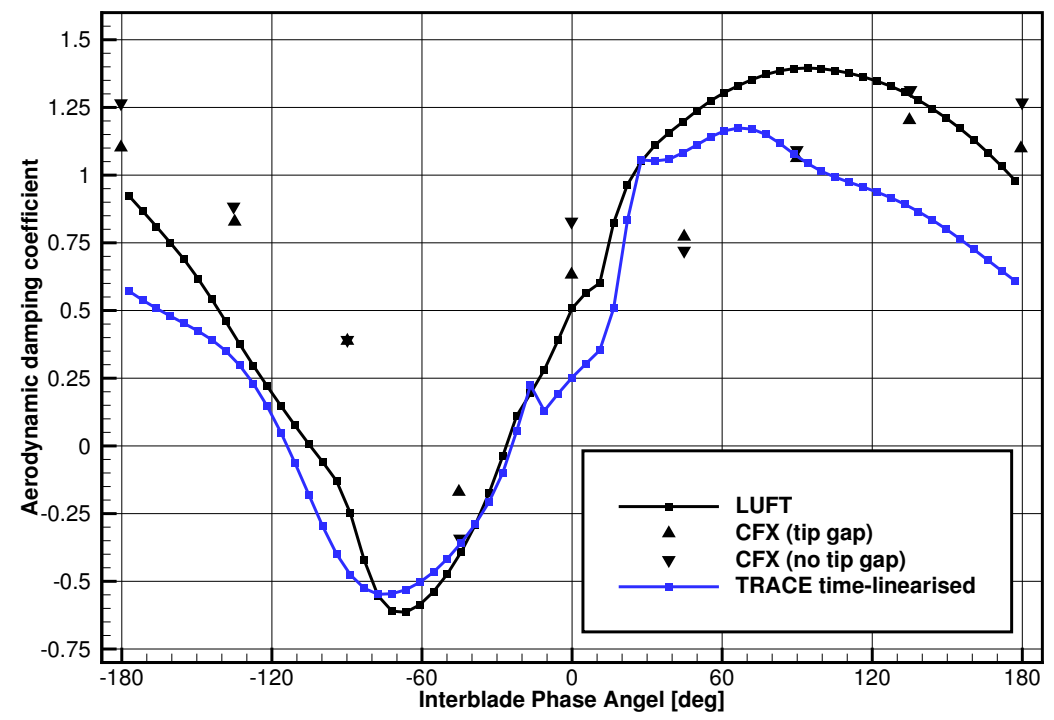

Figure 6. Aerodynamic work coefficient predicted by linearTRACE in comparison with results published by Sun et al. [8].

\subsection{Harmonic Balance and Time-Domain Results}

Figure 7 shows results for the harmonic balance simulations and a time-domain simulation. The time-domain solver was run with the BDF2 integration scheme and 128 time steps per period. The agreement between harmonic balance and unsteady results is very good. To analyse the discrepancies between the time-linearised and the nonlinear methods, a further time-lineariseed simulation was run, which is marked "mod bcs" in the plot, and where the 2D NRBCs were applied to the rotational surface spanned by the interface normals. The standard option, which was used for the damping curve, was to use the circumferential average velocity to define a stream surface, in which the 2D NRBCs were applied. Since this option turned out to be less robust for setups where the radial flow angle was part of the solution, this was not the default in the HB and time-domain solvers. The results 
with consistent boundary conditions explain the apparent offset between the flutter curve and the nonlinear solver results.

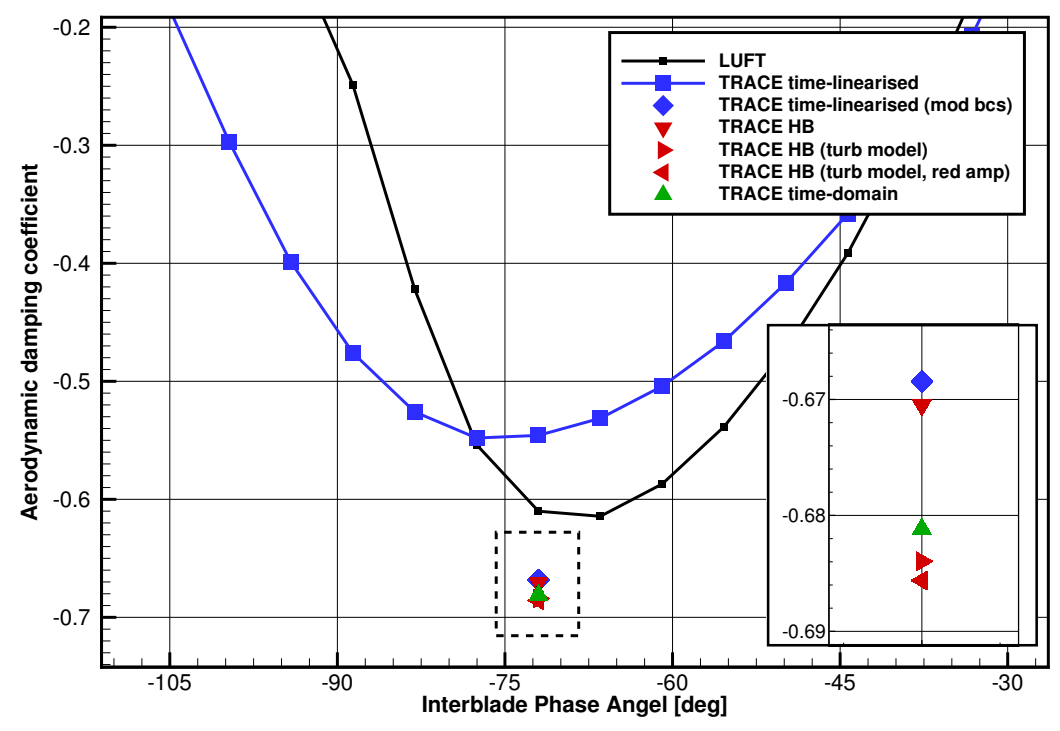

Figure 7. Aerodynamic work coefficient predicted by different harmonic balance set-ups $\sigma=-72^{\circ}$.

The result corresponding to "HB" was obtained with the zeroth and first harmonics but with turbulence quantities modelled as steady, i.e., without higher harmonics. Simulations without the zeroth harmonic turned out to be unstable. The results show a high agreement with the time-linearised solver. The second and third HB simulations correspond to setups with three higher harmonics for both the flow solution and the turbulence quantities $k$ and $\omega$. These results, in turn, were very close to the time-domain results. To analyse whether this was due to nonlinearities, the displacement amplitude was reduced by a factor of 4 . However, the offset between the results with and without harmonics for the turbulence model did not depend greatly on the displacement amplitudes. It was therefore concluded that the difference is mainly due to the unsteadiness in the turbulence model being taken into account.

Figures 8 and 9 show the local work coefficient, i.e., the non-dimensionalised aerodynamic work per cycle and area

$$
w=\frac{\operatorname{Re} d W_{\mathrm{cyc}} / d A}{\pi \alpha^{2} c_{\mathrm{m}} p_{\text {dyn,in }}}
$$

on the upper part of the suction and pressure sides for the three approaches. Here, the HB result is that with the original displacement amplitude but with harmonics for the turbulence quantities. The plot shows that, for $\sigma=-72^{\circ}$, the shock oscillations were responsible for the negative damping. In fact, the local excitation was very strong in the area near $50 \%$ chord on the suction side, where one of the trailing shocks impinges (cf. Figure 3). Note that a different colour map was used for the pressure side since the overall level in aerodynamic work per cycle was much smaller there. The time-linearised result agrees well with the nonlinear results, although greater discrepancies could be observed on the suction side near the leading edge. This was confirmed by Figure 10 where the local damping coefficient is plotted along blade sections at midspan and $90 \%$ channel height. Here, the HB results with steady turbulence modelling are included. At midspan, near 0.65 relative chord, one can see that the turbulence model had an impact on the pressure amplitudes. The HB results coincide either with the time-linearised results or the time-domain results, depending on how the turbulence model was treated. At 90\%, in particular near the leading edge, large discrepancies between linear and non-linear results are visible. 

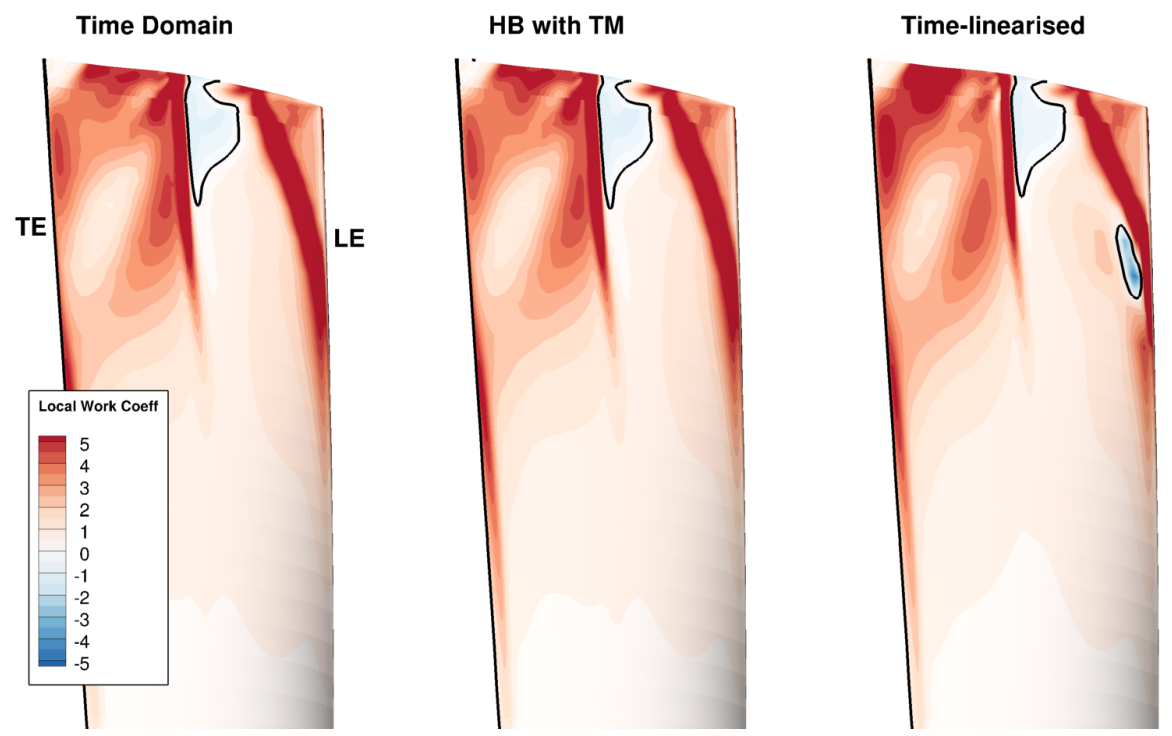

Figure 8. Local work coefficient for $\sigma=-72^{\circ}$ on the upper half of the suction side, predicted by time-domain, harmonic balance, and time-linearised solvers. Black lines mark the boundaries between stable and unstable areas.
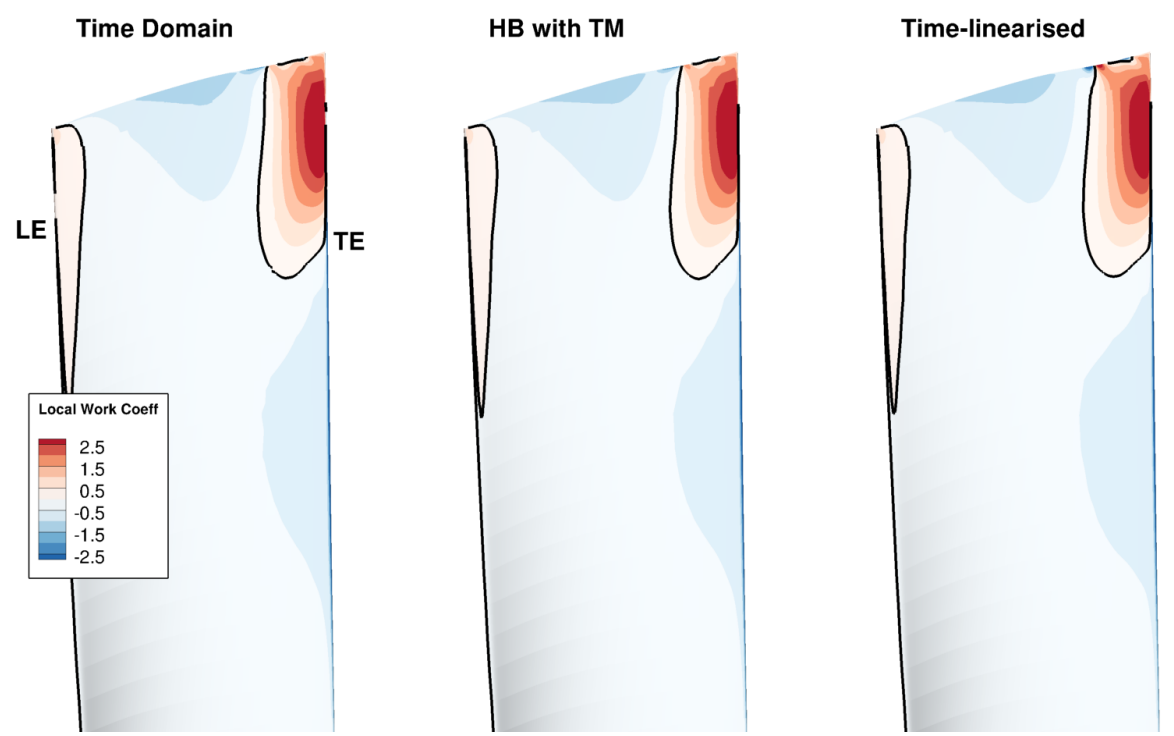

Figure 9. Local work coefficient for $\sigma=-72^{\circ}$ on the upper half of the pressure side, predicted by time-domain, harmonic balance, and time-linearised solvers. Black lines mark the boundaries between stable and unstable areas.

In Figure 11, the convergence of the relative error, again based on current and final values, are plotted for the nonlinear methods. To achieve values within a tolerance of $10^{-3}$, it took about 3500 pseudo time steps for the harmonic balance setups, whereas the time-domain solver had to be run for about 80 periods. This corresponded to about $7700 \mathrm{CPUh}$ for the time-domain simulations, compared to $116 \mathrm{CPUh}$ for the $\mathrm{HB}$ simulation with one higher harmonic and $421 \mathrm{CPUh}$ for the HB setup with three harmonics and higher harmonics for the turbulence model. For the $-72^{\circ}$ interblade phase angle, the linear solver took roughly $61 \mathrm{CPUh}$. It should be noted that in practice a significantly higher error tolerance which would result in smaller CPU times may be acceptable. 

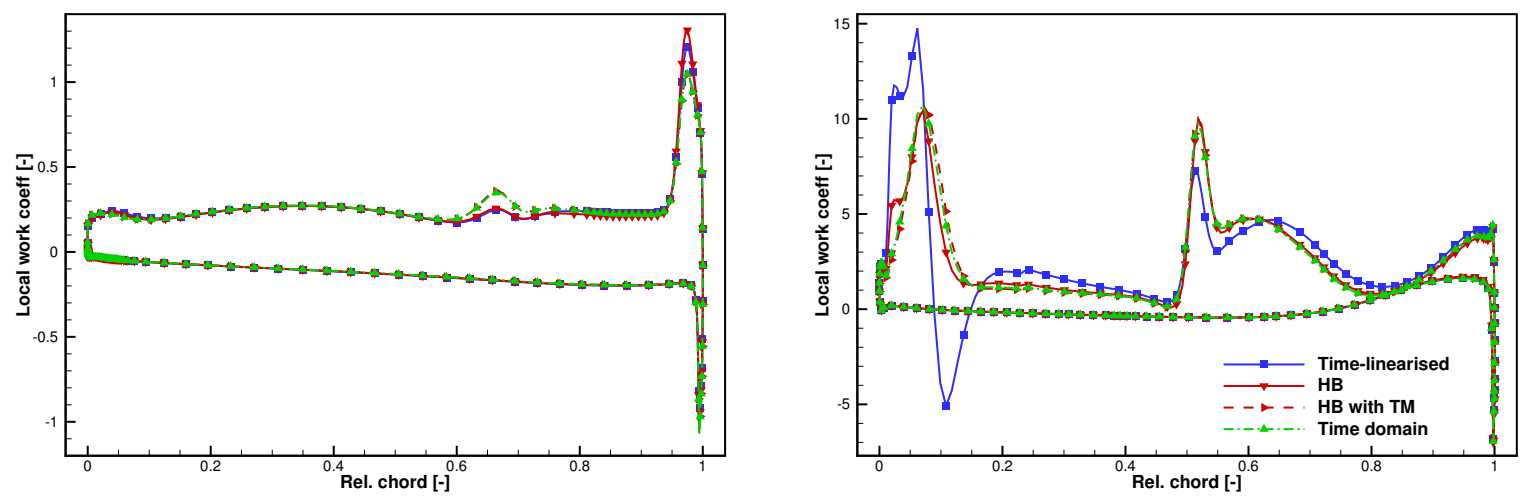

Figure 10. Local work coefficient for $\sigma=-72^{\circ}$ predicted by time-domain, harmonic balance, and timelinearised solvers at 50\% (left) and 90\% (right) channel height.

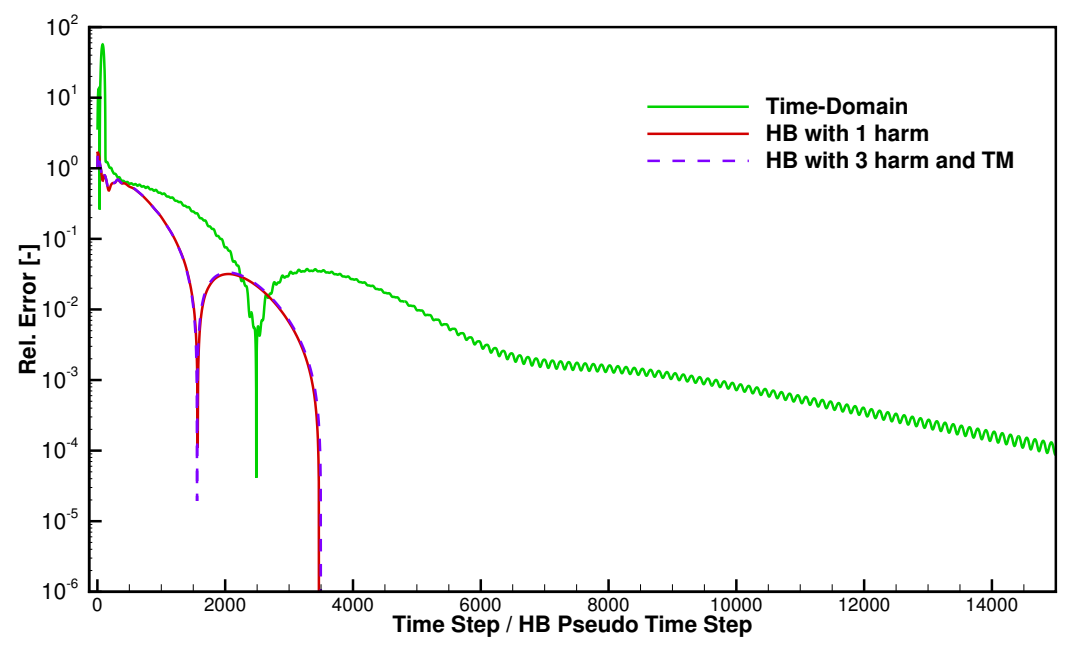

Figure 11. Relative error of aerodynamic work coefficient over time step (time-domain) and pseudo time step (HB) for $\sigma=-72^{\circ}$.

\section{Conclusions}

In this work, time-linearised, harmonic balance and time-integration methods were applied to the flutter analysis of a steam turbine rotor. Since the computational domains, the meshes, and the spatial discretisations were identical, differences in the results can be attributed to the modelling approaches. The results show very good agreement among each other. Differences could be observed that stem from a slight difference in the formulation of the non-reflecting boundary condition and, to a lesser degree though, from the treatment of the turbulence model. Due to the relatively small computational times, the harmonic balance solver is particularly appropriate to study the impact of different modelling approaches. In terms of the overall aerodynamic damping, all results are in good agreement with those obtained by researchers from KTH with the flow solver LUFT.

Author Contributions: Conceptualisation, C.F. and D.S.; data curation, C.F. and D.S.; methodology, C.F., G.A., H.-P.K. and D.S.; software, C.F., G.A., H.-P.K. and D.S.; validation, C.F., G.A., H.-P.K. and D.S.; investigation, C.F.; writing-original draft preparation, C.F.; writing-review and editing, G.A., H.-P.K. and D.S.; visualisation, C.F. and D.S.; project administration, C.F.; and funding acquisition, C.F.

Funding: This study was performed within the framework of the joint project ECOFLEX that is part of the German research corporation AG-Turbo. The research was supported by the industrial partners Siemens AG, MTU Aero Engines AG, and the German Federal Ministry of Economic Affairs and Energy under grant number 03ET7092B. The APC of this paper was funded by Euroturbo.

Conflicts of Interest: The authors declare no conflict of interest. 


\section{Abbreviations}

The following abbreviations are used in this manuscript:

HB Harmonic Balance

NRBC Nonreflecting Boundary Conditions

\section{Nomenclature}

$b \quad$ rotor blade height

$c_{\mathrm{m}} \quad$ chord at midspan

$d_{\max }$ maximal displacement amplitude

$v_{\text {out }} \quad$ velocity magnitude at outlet

i square root of -1

$p_{\text {dyn }} \quad$ dynamic pressure at rotor inlet

$q \quad$ vector of conservative flow variables

$\hat{q}_{\omega} \quad$ Fourier coefficient of $q$ with respect to the angular frequency $\omega$

$\mathscr{F} \quad$ Fourier transform

$R \quad$ flow residual

$V \quad$ cell volume

$x, \dot{x} \quad$ grid coordinates and velocities

$\alpha \quad$ non-dimensionalised amplitude

$\sigma \quad$ interblade phase angle

$\omega \quad$ angular frequency

\section{References}

1. Rendu, Q.; Philit, M.; Labit, S.; Chassaing, J.C.; Rozenberg, Y.; Aubert, S.; Ferrand, P. Time-Linearized and Harmonic Balance Navier-Stokes Computations of a Transonic Flow over an Oscillating Bump. In Proceedings of the 14th International Symposium on Unsteady Aerodynamics, Aeroacoustics \& Aeroelasticity of Turbomachines ISUAAAT14, Stockholm, Sweden, 8-11 September 2015.

2. Lindquist, D.R. Computation of Unsteady Transonic Flowfields Using Shock Capturing and the Linear Perturbation Euler Equations. Ph.D. Thesis, Department of Aeronautics and Astronautics, Massachusetts Institute of Technology, Cambridge, MA, USA, 1992.

3. Seeley, C.E.; Wakelam, C.; Zhang, X.; Hofer, D.; Ren, W.M. Investigations of Flutter and Aero Damping of a Turbine Blade: Part 1-Experimental Characterization. In Proceedings of the ASME Turbo Expo 2016: Turbomachinery Technical Conference and Exposition, Seoul, Korea, 13-17 June 2016; number 49842, p. V07BT34A024.

4. Ren, W.M.; Seeley, C.E.; Zhang, X.; Mitchell, B.E.; Ju, H. Investigations of Flutter and Aero Damping of a Turbine Blade: Part 2-Numerical Simulations. In Proceedings of the ASME Turbo Expo 2016: Turbomachinery Technical Conference and Exposition, Seoul, Korea, 13-17 June 2016; number 49842, p. V07BT34A025.

5. Schlüß, D.; Frey, C. Time Domain Flutter Simulations of a Steam Turbine Stage Using Spectral 2D Non-reflecting Boundary Conditions. In Proceedings of the 15th International Symposium on Unsteady Aerodynamics, Aeroacoustics and Aeroelasticity of Turbomachines (ISUAAAT), Oxford, UK, 24-27 September 2018.

6. Burton, Z. Analysis of Low Pressure Steam Turbine Diffuser and Exhaust Hood Systems. Ph.D. Thesis, Durham University, Durham, UK, 2014.

7. Qi, D.; Petrie-Repar, P.; Gezork, T.; Sun, T. Establishment of an open 3D steam turbine flutter test case. In Proceedings of the 12th European Conference on Turbomachinery Fluid dynamics \& Thermodynamics, Stockholm, Sweden, 3-7 April 2017.

8. Sun, T.; Petrie-Repar, P.; Qi, D. Investigation of Tip Clearance Flow Effects on an Open 3D Steam Turbine Flutter Test Case. In Proceedings of the ASME TurboExpo 2017, Charlotte, NC, USA, 26-30 June 2017; number 50954, p. V008T29A024.

9. Fuhrer, C.; Vogt, D.M. On the Impact of Simulation Approaches on the Predicted Aerodynamic Damping of a Low Pressure Steam Turbine Rotor. In Proceedings of the ASME TurboExpo 2017, Charlotte, NC, USA, $26-30$ June 2017.

10. Roe, P.L. Approximate Riemann solvers, parameter vectors, and difference schemes. J. Comput. Phys. 1981, 43, 357-372. [CrossRef] 
11. van Leer, B. Towards the ultimate conservative difference scheme. V. A second-order sequel to Godunov's method. J. Comput. Phys. 1979, 32, 101-136. [CrossRef]

12. van Albada, G.D.; van Leer, B.; Roberts, W.W., Jr. A comparative study of computational methods in cosmic gas dynamics. Astron. Astrophys. 1982, 108, 76-84.

13. Harten, A. High Resolution Schemes for Hyperbolic Conservation Laws. J. Comput. Phys. 1983, 49, $357-393$. [CrossRef]

14. Kersken, H.P.; Frey, C.; Voigt, C.; Ashcroft, G. Time-Linearized and Time-Accurate 3D RANS Methods for Aeroelastic Analysis in Turbomachinery. J. Turbomach. 2012, 134, 051024. [CrossRef]

15. Frey, C.; Ashcroft, G.; Kersken, H.P.; Voigt, C. A Harmonic Balance Technique for Multistage Turbomachinery Applications. In Proceedings of the ASME Turbo Expo 2014: Turbine Technical Conference and Exposition, Düsseldorf, Germany, 16-20 June 2014; number 45615, p. V02BT39A005.

16. Ashcroft, G.; Frey, C.; Heitkamp, K.; Weckmüller, C. Advanced Numerical Methods for the Prediction of Tonal Noise in Turbomachinery-Part I: Implicit Runge-Kutta Schemes. J. Turbomach. 2013, 136, 021002. [CrossRef]

17. Ashcroft, G.; Frey, C.; Kersken, H.P. On the Development of a Harmonic Balance Method for Aeroelastic Analysis. In Proceedings of the 6th European Conference on Computational Fluid Dynamics (ECFD VI), Barcelona, Spain, 20-25 July 2014.

18. Wilcox, D.C. Reassessment of the Scale-Determining Equation for Advanced Turbulence Models. AIAA J. 1988, 26, 1299-1310. [CrossRef]

19. Ashcroft, G.; Frey, C.; Kersken, H.P.; Kügeler, E.; Wolfrum, N. On The Simulation Of Unsteady Turbulence And Transition Effects In A Multistage Low Pressure Turbine, Part I: Verification And Validation. In Proceedings of the ASME Turbo Expo 2018 Turbomachinery Technical Conference and Exposition, Oslo, Norway, 11-15 June 2018.

20. Kersken, H.P.; Ashcroft, G.; Frey, C.; Wolfrum, N.; Korte, D. Nonreflecting Boundary Conditions for Aeroelastic analysis in Time and Frequency Domain 3D RANS Solvers. In Proceedings of the ASME Turbo Expo 2014, Düsseldorf, Germany, 16-20 June 2014.

21. Schlüß, D.; Frey, C.; Ashcroft, G. Consistent Non-reflecting Boundary Conditions For Both Steady and Unsteady Flow Simulations In Turbomachinery Applications. In Proceedings of the ECCOMAS Congress 2016 VII European Congress on Computational Methods in Applied Sciences and Engineering, Crete Island, Greece, 5-10 June 2016.

22. Petrie-Repar, P.; Makhnov, V.; Shabrov, N.; Smirnov, E.; Galaev, S.; Eliseev, K. Advanced Flutter Analysis of a Long Shrouded Steam Turbine Blade. In Proceedings of the ASME Turbo Expo 2014: Turbine Technical Conference and Exposition. American Society of Mechanical Engineers, Dusseldorf, Germany, 16-20 June 2014; p. V07BT35A022.

23. Weber, A.; Sauer, M. PyMesh-Template Documentation. In Technical Report DLR-IB-AT-KP-2016-34, German Aerospace Center (DLR); Institute of Propulsion Technology: Linder Hoehe, Cologne, Germany, 2016.

24. Department of Energy Technology, KTH Royal Institute of Technology, Stockholm, Sweden. 3D Steam Turbine Flutter Test Case, 2018. Available online: https:/ / www.kth.se/en/itm/inst/energiteknik/forskning/kraftvarme/ekv-researchgroups/turbomachinery-group/aeromech-test-cases/3d-steam-turbine-flutter-testcase-1.706654 (accessed on 18 August 2018).

25. Frey, C.; Kersken, H.P. On the Regularisation of Non-reflecting Boundary Conditions near Acoustic Resonance. In Proceedings of the ECCOMAS Congress 2016 VII European Congress on Computational Methods in Applied Sciences and Engineering, Crete Island, Greece, 5-10 June 2016.

26. Petrie-Repar, P.J.; McGhee, A.M.; Jacobs, P.A. Three-Dimensional Viscous Flutter Analysis of Standard Configuration 10. In Proceedings of the ASME Turbo Expo 2007, Montreal, QC, Canada, 14-17 May 2007.

(c) 2019 by the authors. Licensee MDPI, Basel, Switzerland. This article is an open access article distributed under the terms and conditions of the Creative Commons Attribution NonCommercial NoDerivatives (CC BY-NC-ND) license (https://creativecommons.org/licenses/by-nc-nd/4.0/). 